\title{
Development of DNA markers for the selection of spring barley varieties that are resistant to spot blotch
}

\author{
Rozanova I.V.1,2*, Lashina N.M. ${ }^{3}$, Efimov V.M. ${ }^{2}$, Afanasenko O.S. ${ }^{3}$, \\ Khlestkina E.K. ${ }^{1,2}$ \\ ${ }^{1}$ N.I. Vavilov All-Russian Research Institute of Plant Genetic Resources (VIR), St. Petersburg, Russia \\ ${ }^{2}$ Institute of Cytology and Genetics, SB RAS, Novosibirsk, Russia \\ ${ }^{3}$ All-Russian Research Institute for Plant Protection, St. Petersburg, Russia \\ * email: i.rozanova@vir.nw.ru
}

Spot blotch, caused by Cochliobolus sativus, is the one of the most widespread and harmful diseases in barley. The identification of genetic loci associated with resistance to $C$. sativus is the important task for future marker-assisted selection. The goal of the current study was to identify loci conferring seedling resistance to different pathotypes of $C$. sativus in the Siberian spring barley core collection. The collection included 94 spring barley cultivars and lines was created. All of them were phenotyped at the seedling stage with three $C$. sativus isolates $(\mathrm{O} 2.18, \mathrm{Kr} 2$ and $\mathrm{Ch} 3)$. About $40 \%$ genotypes were resistant to spot blotch. A total of 94 genotypes were analyzed with the barley $50 \mathrm{~K}$ Illumina Infinium iSELECT assay. 27,319 SNPs from total 44,040 SNPs passed filtering threshold and were used for association mapping. Several statistical models were used (GLM, GLM+Q, GLM+PCA, MLM) and they allowed to reveal four genome loci (on chromosome 1H (50-61.2 cM), 2H (68.7-69.68 cM), 3H (18.72-26.18 cM), 7H (7.52$15.44 \mathrm{cM})$ ). Only one locus on chromosome $3 \mathrm{H}$ were revealed using MLM analysis. In any way, further, 12 SNPs from all revealed regions were converted to KASP-markers and validated on independent sample that consist of 11 high resistant and 11 high susceptible varieties. According to obtained data, 5 markers located on chromosome $3 \mathrm{H}$ (18.72-26.18 cM) were determined to possess good accuracy. The data were assessed using PASS, Tassel 5, R and SNP-viewer. Information of SNPs related can be used further for development of DNA-markers convenient for diagnostics of resistanceassociated alleles in barley breeding programs. 